\title{
Asset Demand Based Tests of Expected Utility Maximization
}

\author{
Kübler, Felix ; Selden, Larry ; Wei, Xiao
}

\begin{abstract}
In the neoclassical model of consumer behavior, considerable work has been done investigating when a consumer's demand behavior can be described as having been derived from utility maximization. However, most discussions are in a certainty world. We expand on prior analyses in an uncertainty setting by providing conditions under which contingent claim and asset demands will be consistent with state independent Expected Utility maximization. The question is addressed using two different traditional approaches. First given the analytical form of the demand functions, we derive necessary and sufficient conditions such that the consumer's behavior can be rationalized by an Expected Utility function. Second, we provide a necessary and sufficient condition for a finite set of observations on prices, probabilities and quantities to be consistent with Expected Utility maximization for the case of a single commodity in each state. This condition is shown to be analogous to the strong axiom of revealed preference in classical certainty demand theory. For both approaches, we consider the complete and incomplete asset market cases.
\end{abstract}

DOI: https://doi.org/10.1257/aer.104.11.3459

Posted at the Zurich Open Repository and Archive, University of Zurich ZORA URL: https://doi.org/10.5167/uzh-101280

Journal Article

Originally published at:

Kübler, Felix; Selden, Larry; Wei, Xiao (2014). Asset Demand Based Tests of Expected Utility Maximization. American Economic Review, 104(11):3459-3480.

DOI: https://doi.org/10.1257/aer.104.11.3459 


\section{Asset Demand Based Tests of Expected Utility Maximization*}

\author{
Felix Kubler \\ University of Zurich \\ Swiss Finance Institute
}

\author{
Larry Selden \\ Columbia University \\ University of Pennsylvania
}

Xiao Wei

University of Pennsylvania

January 27, 2014 


\section{Introduction}

The neoclassical certainty model of consumer behavior postulates that a consumer's demand can be described as having been derived from utility maximization subject to a budget constraint. One is then naturally led to ask what this model implies about observed demand behavior. This question has been addressed using two quite distinct approaches. The first, originating in the work of Slutsky (1915) and Antonelli (1886), derives necessary and sufficient conditions for utility maximization assuming knowledge of the consumer's demand functions. The second approach known as "revealed preference", following the classic work of Samuelson (1938), provides necessary and sufficient restrictions on a finite set of demand-price pairs such that the demand behavior of a consumer is consistent with utility maximization.

In this paper, we extend both approaches to a classic portfolio choice problem, where consumers possess state independent Expected Utility preferences and there is a single commodity in each state. We focus only on the complete market case, where the financial asset setting is equivalent to the contingent claim setting and hence it is only necessary to discuss demand tests for the latter. We depart from the standard contingent claim theory by assuming that state of nature probabilities are not fixed and we model demand as a function of probabilities as well as prices and income.

For the revealed preference approach, with the restriction of a single commodity in each state, we derive a much simpler test for Expected Utility maximization than those that currently exist in the literature. For example the formulation of Green and Srivastava (1986), as in the revealed preference models of Afriat (1967) and Varian (1983), postulates the existence of unknown utility levels and multipliers. We do not require these existential quantifiers and our condition is analogous to the strong axiom of revealed preference in classical demand theory. The conditions involve probabilities but remain necessary and sufficient when probabilities are fixed (and known). From our revealed preference test, it is possible to tell whether the Expected Utility hypothesis is consistent with the assumed finite data set. If consistency is not refuted and one wants to use the data to estimate a demand function, one requires restrictions on the functional form to be employed which ensure that the estimated function is rationalized by an Expected Utility function.

We derive simple restrictions on contingent claim demand functions which are necessary and sufficient for preferences to be representable by a state independent Expected Utility function. Going back to at least Samuelson (1947), there is a substantial literature on the certainty version of this problem but there are few 
results in the literature that are concerned with contingent claim demand and Expected Utility maximization. Our approach differs from that of Dybvig (1983) in two ways. First, we do not need to assume that the representation is additively separable. Second, we require knowledge of how demand varies with exogenously varying probabilities. Dybvig's test can be conducted on the functional form of demands with or without knowing the explicit values of probabilities. However because for him probabilities are fixed, his test fails to distinguish between demands coming from Expected Utility functions and representations which one would not typically refer to as Expected Utility. ${ }^{1}$

Given the seminal role played by restrictions on the Slutsky matrix in ensuring the existence of a well behaved utility function that rationalizes demand, we investigate what additional Slutsky restrictions correspond to utility taking the Expected Utility form. Goldman and Uzawa (1964) give necessary and sufficient conditions for additively separable utility based on terms in the Slutsky matrix. We simplify their conditions for our case of one good per state and derive two additional conditions, one of which is a new derivative condition involving terms in the Slutsky matrix. Together these three conditions are necessary and sufficient for demand to be rationalized by Expected Utility.

Clearly our assumption that state of nature probabilities vary confronts obvious obstacles in practical implementation. However, the implicit assumption in the standard approach that repeated observations on demands can be obtained while holding probabilities fixed seems less than fully compelling. One would expect that observations on an individual's choices in financial markets where prices change could correspond to the case where probability distributions of asset payoffs also change. To explore these issues, recent new approaches to laboratory experiments may offer some promise for extending classical tests of consumer behavior to models with uncertainty (see, for example, Choi, et al. 2007 and Andreoni and Sprenger 2012).

The rest of the paper is organized as follows. The next section introduces notation and the basic contingent claim/asset optimization problem. In Section 3 , we derive necessary and sufficient conditions for an observed finite set of demand, price and probability triplets to be consistent with Expected Utility maximization. Section 4 provides restrictions on demands such that they are rationalizable by Expected Utility functions.

\footnotetext{
${ }^{1}$ See Example 1 and Remark 3 below.
} 


\section{Preliminaries}

Throughout this paper we consider a classic complete market portfolio choice setting with $S$ states of nature, a single commodity per state and $J=S$ financial assets. Denote the payoff for asset $j(j \in\{1,2, \ldots, J\})$ in state $s(s \in\{1,2, \ldots, S\})$ by $\xi_{j s}$ and the matrix of payoffs by $\Xi$. The quantities of assets are denoted by $z_{j}$ and their prices are denoted by $P_{j}$. An individual has preferences over state contingent consumption, $\mathbf{x} \in \mathbb{R}_{++}^{S}$, that depend on the probabilities $\pi_{1}, \ldots, \pi_{S}$ and is represented by a utility function $U(\mathbf{x} ; \boldsymbol{\pi})$ which is assumed to be strictly quasiconcave, strictly increasing in $\mathbf{x}$ and three times continuously differentiable in $\mathbf{x}$ and $\boldsymbol{\pi}$. It should be noted that $\boldsymbol{\pi}$ enters into $U$ as a parameter that is allowed to vary exogenously, but $\boldsymbol{\pi}$ is not a choice variable. The consumer's preferences are defined over contingent claims. The utility $U(\mathbf{x} ; \boldsymbol{\pi})$ is said to take the state independent Expected Utility form when

$$
U(\mathbf{x} ; \boldsymbol{\pi})=\sum_{s=1}^{S} \pi_{s} u\left(x_{s}\right),
$$

where $u$ denotes the NM (von Neumann-Morgenstern) index and satisfies $u \in C^{3}$, $u^{\prime}>0$ and $u^{\prime \prime}<0$.

Given income $I$ and facing prices $\mathbf{P}$ and probabilities $\boldsymbol{\pi}$, the agent solves

$$
\begin{aligned}
\max _{\mathbf{x} \in \mathbb{R}_{++}^{s}, \mathbf{z} \in \mathbb{R}^{J}} U(\mathbf{x} ; \boldsymbol{\pi}) \quad \text { S.T. } & \mathbf{P} \cdot \mathbf{z} \leq I \\
& x_{s}=\sum_{j=1}^{S} z_{j s} \xi_{j s} \text { for all } s=1, \ldots, S .
\end{aligned}
$$

We investigate what restrictions the assumption of state independent Expected Utility imposes on the solution of this maximization problem as a function of prices, incomes and probabilities. We consider two settings. First we assume that we have $N$ observations on asset demands, prices, as well as probabilities $\left(\mathbf{z}^{i}, \mathbf{P}^{i}, \boldsymbol{\pi}^{i}\right)_{i=1}^{N}$. Second, we assume that we observe the demand function $\mathbf{z}(\mathbf{P}, \boldsymbol{\pi}, I)$ for an open set of prices, incomes and probabilities. Probabilities are assumed to be known. In our demand function tests we investigate when $U$ takes the Expected Utility form (1) for all specifications of $\boldsymbol{\pi}$. Some of our tests will be seen to depend critically on how the probability parameter $\boldsymbol{\pi}$ enters into the consumer's demand function.

Our assumption that markets are complete ensures that the $S$ assets' payoffs are linearly independent. This allows us to transform our observations into $(\mathbf{x}, \mathbf{p}, \boldsymbol{\pi})$, where $\mathbf{x}=\Xi \mathbf{z}$ and $\mathbf{P}^{T}=\mathbf{p}^{T} \Xi$. Therefore, we focus on the contingent 
claim demand $\mathbf{x}(\mathbf{p}, \boldsymbol{\pi}, I)$ that solves

$$
\max _{\mathbf{x} \in \mathbb{R}_{++}^{s}} U(\mathbf{x} ; \boldsymbol{\pi}) \quad S . T . \mathbf{p} \cdot \mathbf{x} \leq I,
$$

where $\mathbf{p}=\left(p_{1}, \ldots, p_{S}\right)$ is the contingent claim price vector. Note that if contingent claim demand is derived from the Expected Utility function (1), demand is homogeneous of degree zero in $\boldsymbol{\pi}$. It is not necessary to normalize probabilities and since we consider derivatives with respect to probabilities, it will prove convenient not to do so. We therefore assume $\boldsymbol{\pi} \in \mathbb{R}_{++}^{S}$.

We next consider an example which highlights our key assumption that demand is a continuous, differentiable function of probabilities as well as prices and income. This differentiates our approach from most contingent claim formulations which assume demand is a function of prices and income, but probabilities are fixed parameters.

Example 1 Assume there are three states of nature and preferences are represented by the following utility

$$
U\left(x_{1}, x_{2}, x_{3} ; \pi_{1}, \pi_{2}, \pi_{3}\right)=-\sum_{s=1}^{3} \pi_{s}\left(\exp \left(-\pi_{1} x_{s}\right)+\exp \left(-\pi_{2} x_{s}\right)+\exp \left(-\pi_{3} x_{s}\right)\right) .
$$

Clearly this representation is not an Expected Utility function since probabilities do not enter into the function linearly. Moreover each subutility $u\left(x_{s}\right)$, although being state independent, is probability dependent which of course is inconsistent with the classic NM index in eqn. (1). Suppose instead of assuming that $\boldsymbol{\pi}$ is an exogenous variable, probabilities take on fixed values as in the standard contingent claim setting (e.g., Dybvig 1983). Then if

$$
\pi_{1}=0.6, \pi_{2}=0.3 \text { and } \pi_{3}=0.1 \text {, }
$$

the utility (4) can be expressed as

$U\left(x_{1}, x_{2}, x_{3} ; \pi_{1}, \pi_{2}, \pi_{3}\right)=-\sum_{s=1}^{3} \pi_{s}\left(\exp \left(-0.6 x_{s}\right)+\exp \left(-0.3 x_{s}\right)+\exp \left(-0.1 x_{s}\right)\right)$.

This can be viewed as taking the state independent Expected Utility form of eqn.

(1) with the NM index given by

$$
u(x)=-(\exp (-0.6 x)+\exp (-0.3 x)+\exp (-0.1 x)) .
$$

If one alternatively assumes that probabilities are given by

$$
\pi_{1}=0.50, \pi_{2}=0.35 \text { and } \pi_{3}=0.15,
$$


once again the form of $U$ can be viewed as taking the state independent Expected Utility form. However now the NM index

$$
u(x)=-(\exp (-0.50 x)+\exp (-0.35 x)+\exp (-0.15 x))
$$

differs from (7) by more than a positive affine transform. Any demand test based on the assumption of fixed probabilities will not be able to distinguish between (4) and (6).

\section{A Revealed Preference Test}

In this section, we provide conditions for a finite set of demand-price-probability triplets which are necessary and sufficient for the demand behavior of a consumer to be consistent with state independent Expected Utility maximization.

Green and Srivastava (1986) derive a complete market revealed preference test of Expected Utility maximization in a setting where there are multiple goods in each state. We consider the simpler case where there is a single good in each state. This will enable us to eliminate the assumption of existential quantifiers required in Green and Srivastava (1986) and obtain a result that is essentially analogous to the strong axiom of revealed preference.

We have $N$ observations on contingent claim demands, prices and probabilities $\left(\mathbf{x}^{i}, \mathbf{p}^{i}, \boldsymbol{\pi}^{i}\right)_{i=1}^{N}$ with $\mathbf{x}^{i} \in \mathbb{R}_{++}^{S}, \mathbf{p}^{i} \in \mathbb{R}_{++}^{S}$ and $\boldsymbol{\pi}^{i}$ being a vector of positive probabilities for each $i=1, \ldots, N$. In line with the rest of the paper, we assume that the probabilities are observable and can vary across observations. ${ }^{2}$ Without loss of generality, we assume throughout this section that $x_{s}^{i} \neq x_{s^{\prime}}^{j}$ for all $i, j$ and all $s, s^{\prime}$ (the case where $x_{s}^{i}=x_{s^{\prime}}^{j}$ for some $i, j$ and some $s, s^{\prime}$ can be easily considered separately). In order to eliminate the assumption of existential quantifiers required in Green and Srivastava (1986), it will prove convenient to define $\rho_{s}^{i}=p_{s}^{i} / \pi_{s}^{i}$ for all $i=1, \ldots, N, s=1, \ldots, S$, and the operator

$$
L(i, j)=\max _{s, s^{\prime}: x_{s}^{i}>x_{s^{\prime}}^{j}} \frac{\rho_{s}^{i}}{\rho_{s^{\prime}}^{j}},
$$

where we set $L(i, j)=0$ if $x_{s}^{i}<x_{s^{\prime}}^{j}$ for all $s, s^{\prime}$.

Definition 1 We say that $\left(\mathbf{x}^{i}, \mathbf{p}^{i}, \boldsymbol{\pi}^{i}\right)_{i=1}^{N}$ satisfy the strong axiom of revealed expected utility (SAREU) if for any $m>1$ and all $i_{1}, \ldots, i_{m} \in\{1, \ldots, N\}$ we have

$$
L\left(i_{1}, i_{2}\right) \cdot L\left(i_{2}, i_{3}\right) \cdot \ldots \cdot L\left(i_{m-1}, i_{m}\right) \cdot L\left(i_{m}, i_{1}\right)<1 .
$$

\footnotetext{
${ }^{2}$ If probabilities are not observable our conditions in Theorem 1 below remain necessary and sufficient if one treats probabilities as unknown positive numbers and condition (ii) is adjusted to say that there exist real numbers $\pi_{s}^{i}>0$ and $\lambda^{i}>0$.
} 
Remark 1 Note that the case where $m=2$ and $i_{1}=i_{2}=i$ implies that $L(i, i)<$ 1 , which is equivalent to

$$
x_{s}^{i}>x_{s^{\prime}}^{i} \Rightarrow \rho_{s}^{i}<\rho_{s^{\prime}}^{i}
$$

for each $i$ and all $s, s^{\prime}$.

Comparing our analysis with the certainty formulation of Afriat (1967), it can be observed that SAREU plays the role of Afriat's cyclic consistency which as Varian (1982) argues is a version of the general axiom of revealed preference (GARP). As in Afriat, our condition (11) must hold for all cycles. However our condition involves the product of the risk neutral prices (i.e., $\left.\frac{p_{s}}{\pi_{s}}\right)$ rather than a sequence of price-quantity inequalities.

We have the following Theorem.

Theorem 1 The following three statements are equivalent.

(i) There exists a continuous, strictly increasing and strictly concave $N M$ index $u: \mathbb{R}_{++} \rightarrow \mathbb{R}$ such that for all $i=1, \ldots, N$

$$
\mathbf{x}^{i} \in \underset{\mathbf{x} \in \mathbb{R}_{++}^{S}}{\arg \max } \sum_{s=1}^{S} \pi_{s}^{i} u\left(x_{s}\right) \quad S . T \cdot \mathbf{p}^{i} \cdot \mathbf{x} \leq \mathbf{p}^{i} \cdot \mathbf{x}^{i} .
$$

(ii) There exist real numbers $\lambda^{i}>0$ for $i=1, \ldots, N$ such that for all $i, j=1, \ldots, N$ and any $s, s^{\prime}=1, \ldots, S$,

$$
x_{s}^{i}>x_{s^{\prime}}^{j} \Rightarrow \lambda^{i} \rho_{s}^{i}<\lambda^{j} \rho_{s^{\prime}}^{j} .
$$

(iii) The observations $\left(\mathbf{x}^{i}, \mathbf{p}^{i}, \boldsymbol{\pi}^{i}\right)_{i=1}^{N}$ satisfy SAREU.

Proof. The equivalence between (i) and (ii) is standard, see e.g., Kubler and Schmedders (2010), Lemma 2. To show the equivalence of (ii) and (iii), we first show that (ii) implies (iii). From (ii), for $i=j$, clearly we have

$$
x_{s}^{i}>x_{s^{\prime}}^{i} \Rightarrow \lambda^{i} \rho_{s}^{i}<\lambda^{i} \rho_{s^{\prime}}^{i} \Rightarrow \rho_{s}^{i}<\rho_{s^{\prime}}^{i},
$$

implying that SAREU holds for this case. For all $i \neq j$ if $x_{s}^{i}>x_{s^{\prime}}^{j}$, we must have that $\frac{\lambda^{j}}{\lambda^{i}}>L(i, j)$. Since $\frac{\lambda^{i_{2}}}{\lambda^{i_{1}}} \cdot \frac{\lambda^{i_{3}}}{\lambda^{i_{2}}} \ldots \cdot \frac{\lambda^{i_{1}}}{\lambda^{i_{m}}}=1$, we must have that eqn. (11) of SAREU holds.

Finally we show that (iii) implies (ii). Given that SAREU holds, and given $N$ observations, there must be an $\epsilon>0$ such that for any $m>1$ all $i_{1}, \ldots, i_{m} \in$ $\{1, \ldots, N\}$ we have

$$
L\left(i_{1}, i_{2}\right) \cdot L\left(i_{2}, i_{3}\right) \cdot \ldots \cdot L\left(i_{m-1}, i_{m}\right) \cdot L\left(i_{m}, i_{1}\right)<\frac{1}{(1+\epsilon)^{m}} .
$$


We can then construct $\left(\lambda^{i}\right)_{i=1}^{N}$ as follows. First without loss of generality we can take $\mathbf{x}^{1}$ from the first observation to contain the largest consumption value, i.e., there is an $s$ so that $x_{s}^{1}>x_{s^{\prime}}^{i}$ for all $i, s^{\prime}$. Then define $\lambda^{1}=1$ and for $j=2,3, \ldots, N$,

$$
\lambda^{j}=\max _{k} \max _{\left(1, i_{2}, \ldots, i_{k-1}, j\right) \in \mathcal{I}_{k}} L\left(1, i_{2}\right) \cdot \ldots \cdot L\left(i_{k-1}, j\right)(1+\epsilon)^{k}
$$

where $\mathcal{I}_{k}=\left\{\left(i_{1}, i_{2}, \ldots, i_{k-1}, i_{k}\right) \mid i_{m} \in\{1,2, \ldots, N\}\right.$ for each $\left.m=1, \ldots, k\right\}$. Note that eqn. (16) and the assumption that $x_{s}^{1}$ is the largest consumption value for some $s$ guarantee that each $\lambda^{j}$ is positive and well defined. In effect the maximum is only taken over vectors of at most length $N$, since eqn. (16) implies that cycles where $i_{l}=i_{k}$ for some $l, k$ can never be part of the maximizing sequence of $L(i, j)$. To prove that (iii) implies (ii), it suffices to show that $\lambda^{i}>\lambda^{j} \frac{\rho_{s}^{j}}{\rho_{s^{\prime}}^{i}}$ whenever $x_{s}^{j}>x_{s^{\prime}}^{i}$. Note that there must be some $k$ and some $i_{2}, \ldots, i_{k-1}$ so that $\lambda^{j}=L\left(1, i_{2}\right) \cdot \ldots \cdot L\left(i_{k-1}, j\right)(1+\epsilon)^{k}$. But by the definition of $L(j, i)$, we have

$$
\lambda^{i}>L\left(1, i_{2}\right) \ldots L\left(i_{k-1}, j\right)(1+\epsilon)^{k} \cdot L(j, i) \geq \lambda^{j} \frac{\rho_{s}^{j}}{\rho_{s^{\prime}}^{i}} .
$$

This proves the Theorem.

Remark 2 Note that in Theorem 1 (i) we only require the NM index $u$ to be continuous and concave and do not require $u \in C^{3}$ as assumed in Section 2. However it follows from Chiappori and Rochet (1987) that with a finite number of observations, it is irrelevant whether one assumes differentiability or not. In particular, their Theorem ( $p$. 688) implies that one can take $u \in C^{\infty}$ since for any finite set of observations satisfying SAREU there exists an infinitely differentiable utility function that rationalizes these observations.

Of course, Theorem 1 holds for fixed probabilities and is then comparable to Theorem 1 in Green and Srivastava (1986). It follows that although Green and Srivastava only consider a fixed probability setting, actually their analysis can naturally be extended to the case when state probabilities are allowed to change. Conditions (i) and (ii) of Theorem 1 are then essentially the same as conditions (i) and (ii) of Theorem 1 in Green and Srivastava (1986). They show that (i) is equivalent to the existence of $\left(u_{s}^{i}\right)_{i=1 \ldots N, s=1 \ldots S}$ and $\left(\lambda^{i}\right)_{i=1}^{N}$ such that

$$
u_{s^{\prime}}^{j}-u_{s}^{i} \leq \lambda^{i} \rho_{s}^{i}\left(x_{s^{\prime}}^{j}-x_{s}^{i}\right) \text { for all } i, j, s, s^{\prime}
$$

with the strict inequality holding in our one commodity case, whenever $x_{s^{\prime}}^{j} \neq x_{s}^{i}$. Adding up the inequalities for $j, s^{\prime}$ and $i, s$ and the one for $i, s$ and $j, s^{\prime}$, we obtain

$$
0 \leq \lambda^{i} \rho_{s}^{i}\left(x_{s^{\prime}}^{j}-x_{s}^{i}\right)+\lambda^{j} \rho_{s}^{j}\left(x_{s}^{i}-x_{s^{\prime}}^{j}\right)
$$


which is obviously equivalent to (ii) if we assume that $x_{s}^{i} \neq x_{s^{\prime}}^{j}$. Since the $x_{s}^{i}$ are scalars, the converse is also true. This is easily seen by observing that given $x_{s}^{i}, \rho_{s}^{i}$ and $\lambda^{i}$ for all $i, s$, we can construct a piecewise linear and concave utility function where $\lambda^{i} \rho_{s}^{i}$ is taken to be the slope at $x_{s}^{i}$.

Since we impose the more restrictive assumption that there is only one good in each state, we are able to derive the new result Theorem 1(iii), which does not require the existential quantifiers $\left(\lambda^{i}\right)_{i=1}^{N}$. In this sense, Theorem 1(iii) is analogous to Afriat's cyclic consistency and is analogous to the strong axiom of revealed preference.

Varian (1983) derives a revealed preference condition for asset demand in a setting with possibly incomplete markets and a single commodity. For the case of complete markets Theorem 1 in his paper becomes the same as eqn. (19) above. As in Green and Srivastava (1986) all his conditions involve unknown multipliers and utility levels.

It follows from Varian (1988) that a necessary and sufficient condition for an Expected Utility function to exist that rationalizes one demand observation $(\mathbf{x}, \mathbf{p}, \boldsymbol{\pi})$ is that $x_{s}$ is a decreasing function of $p_{s} / \pi_{s}$. This is equivalent to Theorem 1(ii) when $i=j$. The contribution of our result is to show the equivalence between (ii) and our new condition SAREU (iii) for any finite number of observations.

Two interesting, recent papers focus on revealed preference tests for Expected Utility preferences. Echenique and Saito (2013) develop a revealed preference test for (Savage) subjective Expected Utility preferences and probabilities. They show that our SAREU is similar to their revealed preference axiom if probabilities become observable in their framework. Polisson and Quah (2013) provide a new procedure for testing the consistency of observed contingent claim demands with maximizing general uncertainty preferences without assuming concavity.

\section{Demand Function Tests}

Suppose the consistency test for Expected Utility preferences based on a finite data set discussed in the prior section is not refuted and one wants to use the data to estimate a demand function. Then it is necessary to derive restrictions on the functional form to be employed in the test. In this section, we derive two different sets of restrictions on the demand functions which are necessary and sufficient for preferences to be representable by an Expected Utility function. The Slutsky matrix for any given demands (asset or contingent claim) is assumed to be symmetric and negative semidefinite, implying that the demands can always be rationalized by a utility function. The question is whether it takes the Expected 
Utility form.

\subsection{Functional Form Restrictions}

The following characterizes restrictions on the form of the demand functions $\mathbf{x}(\mathbf{p}, \boldsymbol{\pi}, I)$ which are necessary and sufficient for the generating utility to be ordinally equivalent to a state independent Expected Utility function. ${ }^{3}$

Theorem 2 Assume that $S>2$ and contingent claim demands $x_{s}(\mathbf{p}, \boldsymbol{\pi}, I)>0$ $(s=1,2, \ldots, S)$ can be rationalized by a well defined utility function. Then this utility function is ordinally equivalent to a state independent Expected Utility function as defined in eqn. (1) if and only if there is a twice continuously differentiable function $f: \mathbb{R}_{++}^{2} \rightarrow \mathbb{R}_{++}$such that the contingent claim demands $x_{s}(\mathbf{p}, \boldsymbol{\pi}, I)$ $(s=1,2, \ldots, S)$ satisfy $x_{s}=f\left(x_{1}, k_{s}\right)$, where $k_{s}$ is defined by

$$
k_{s}={ }_{d e f} \frac{\pi_{s} p_{1}}{\pi_{1} p_{s}},
$$

$f\left(x_{1}, k_{s}\right)$ is strictly increasing in $k_{s}$ and $f(x, 1)=x$ for all $x$.

To prove this result, we will utilize a modified version Samuelson's (1947) classic necessary and sufficient condition for additive separable utility (in a certainty setting).

Lemma 1 When $S>2$, preferences are representable by a twice continuously differentiable additively separable utility

$$
U(\mathbf{x})=\sum_{s=1}^{S} u_{s}\left(x_{s}\right)
$$

if and only if

$$
\frac{\partial\left(\frac{\partial U / \partial x_{s}}{\partial U / \partial x_{1}}\right)}{\partial x_{i}}=0 \quad(\forall i, s \in\{2,3, \ldots, S\}, i \neq s) .
$$

\footnotetext{
${ }^{3}$ Relative to the result in Theorem 2, it should be noted that Andreoni and Sprenger (2012) observe that in a two period discounted Expected Utility (DEU) model, intertemporal allocations will depend only on the ratio of the intertemporal probabilities $\pi_{1} / \pi_{2}$ and not on $\pi_{1}$ or $\pi_{2}$ separately, where $\pi_{1}$ and $\pi_{2}$ are the probabilities of the outcomes with nonzero utility value in periods one and two, respectively. They point out that this is a critical and testable implication of the DEU model. This conclusion uses a similar idea to eqn. (21) in Theorem 2, although their setting is very different from ours.
} 
It should be emphasized that Samuelson (1947, p. 179) actually considers conditions on the inverse demand function that ensure existence of a separable utility and that he does not assume the existence of $U$ in his discussion. In his framework, the the marginal rate of substitution in (23) is replaced by the the inverse demand function

$$
\frac{\partial U / \partial x_{i}}{\partial U / \partial x_{j}}=\frac{p_{i}}{p_{j}}
$$

and he requires that, in addition to (23) the following holds

$$
\frac{\partial\left(\frac{\partial U / \partial x_{s}}{\partial U / \partial x_{2}}\right)}{\partial x_{1}}=0 \quad(\forall s \in\{3,4, \ldots, S\}) .
$$

If one does not start with inverse demand, but assumes that $U$ exists, then (23) implies (25). To see this, notice that $\forall s \in\{3,4, \ldots, S\}$,

$$
\frac{\partial\left(\frac{\partial U / \partial x_{2}}{\partial U / \partial x_{1}}\right)}{\partial x_{s}}=0 \Leftrightarrow \frac{\partial^{2} U}{\partial x_{2} \partial x_{s}} \frac{\partial U}{\partial x_{1}}-\frac{\partial^{2} U}{\partial x_{1} \partial x_{s}} \frac{\partial U}{\partial x_{2}}=0
$$

and

$$
\frac{\partial\left(\frac{\partial U / \partial x_{s}}{\partial U / \partial x_{1}}\right)}{\partial x_{2}}=0 \Leftrightarrow \frac{\partial^{2} U}{\partial x_{2} \partial x_{s}} \frac{\partial U}{\partial x_{1}}-\frac{\partial^{2} U}{\partial x_{1} \partial x_{2}} \frac{\partial U}{\partial x_{s}}=0 .
$$

Subtracting the above two equations yields (25)

$$
\frac{\partial^{2} U}{\partial x_{1} \partial x_{s}} \frac{\partial U}{\partial x_{2}}-\frac{\partial^{2} U}{\partial x_{1} \partial x_{2}} \frac{\partial U}{\partial x_{s}}=0 \Leftrightarrow \frac{\partial}{\partial x_{1}}\left(\frac{\partial U / \partial x_{s}}{\partial U / \partial x_{2}}\right)=0 .
$$

Next we will give the proof of Theorem 2 .

Proof. Necessity follows directly from the first order condition. Next we prove sufficiency. We first show that for $S>2$, the condition $x_{s}=f\left(x_{1}, k_{s}\right)$ implies that $U$ is (ordinally) additively separable. Given the existence of $U$, the first order conditions for the maximization (3) are

$$
\frac{\partial U / \partial x_{1}}{\partial U / \partial x_{s}}=\frac{p_{1}}{p_{s}} \quad(s=2,3, \ldots, S) .
$$

For each $s \in\{2, \ldots, S\}$, since $x_{s}=f\left(x_{1}, k_{s}\right)$ and $f\left(x_{1}, k_{s}\right)$ is a strictly increasing function of $k_{s}$, we have

$$
\frac{\pi_{s} p_{1}}{\pi_{1} p_{s}}=k_{s}=f_{x_{1}}^{-1}\left(x_{s}\right)
$$

or equivalently

$$
\frac{\partial U / \partial x_{s}}{\partial U / \partial x_{1}}=\frac{\pi_{s}}{\pi_{1} f_{x_{1}}^{-1}\left(x_{s}\right)},
$$

implying that

$$
\frac{\partial\left(\frac{\partial U / \partial x_{s}}{\partial U / \partial x_{1}}\right)}{\partial x_{i}}=0(\forall i, s \in\{2,3, \ldots, S\}, i \neq s) .
$$


When $S>2$, (32) is obviously identical to eqn. (23) in Lemma 1. Thus it follows from Lemma 1 that one can assume $U$ takes the form

$$
U(\mathbf{x} ; \boldsymbol{\pi})=\sum_{s=1}^{S} u_{s}\left(x_{s} ; \boldsymbol{\pi}\right) .
$$

Therefore the first order condition can be written as

$$
\frac{u_{1}^{\prime}\left(x_{1} ; \boldsymbol{\pi}\right)}{u_{s}^{\prime}\left(x_{s} ; \boldsymbol{\pi}\right)}=\frac{p_{1}}{p_{s}} \Leftrightarrow \frac{\pi_{s} u_{1}^{\prime}\left(x_{1} ; \boldsymbol{\pi}\right)}{\pi_{1} u_{s}^{\prime}\left(x_{s} ; \boldsymbol{\pi}\right)}=k_{s} .
$$

Since $x_{s}=f\left(x_{1}, k_{s}\right)$, we have $k_{s}=f_{x_{1}}^{-1}\left(x_{s}\right)$, implying that

$$
\frac{\pi_{s} u_{1}^{\prime}\left(x_{1} ; \boldsymbol{\pi}\right)}{\pi_{1} u_{s}^{\prime}\left(x_{s} ; \boldsymbol{\pi}\right)}=f_{x_{1}}^{-1}\left(x_{s}\right)
$$

must be independent of $\boldsymbol{\pi}$. Denoting

$$
h_{s}\left(x_{s} ; \boldsymbol{\pi}\right)=\frac{u_{s}^{\prime}\left(x_{s} ; \boldsymbol{\pi}\right)}{\pi_{s}},
$$

we have

$$
\frac{\partial}{\partial \boldsymbol{\pi}}\left(\frac{h_{1}\left(x_{1} ; \boldsymbol{\pi}\right)}{h_{s}\left(x_{s} ; \boldsymbol{\pi}\right)}\right)=0
$$

implying that

$$
\frac{\partial\left(\ln h_{1}\left(x_{1} ; \boldsymbol{\pi}\right)\right)}{\partial \boldsymbol{\pi}}=\frac{\partial\left(\ln h_{s}\left(x_{s} ; \boldsymbol{\pi}\right)\right)}{\partial \boldsymbol{\pi}} .
$$

Taking the derivative with respect to $x_{s}$ on both sides of the above equation yields

$$
\frac{\partial^{2}\left(\ln h_{s}\left(x_{s} ; \boldsymbol{\pi}\right)\right)}{\partial x_{s} \partial \boldsymbol{\pi}}=0
$$

implying that

$$
\ln h_{s}\left(x_{s} ; \boldsymbol{\pi}\right)=G_{s}\left(x_{s}\right)+H_{s}(\boldsymbol{\pi}),
$$

where $H_{s}(\boldsymbol{\pi})$ is a function of probabilities and $G_{s}\left(x_{s}\right)$ is a function of $x_{s}$. Defining

$$
K_{s}(\boldsymbol{\pi})=\exp \left(H_{s}(\boldsymbol{\pi})\right) \text { and } v_{s}\left(x_{s}\right)=\exp \left(G_{s}\left(x_{s}\right)\right)
$$

we immediately obtain

$$
h_{s}\left(x_{s} ; \boldsymbol{\pi}\right)=K_{s}(\boldsymbol{\pi}) v_{s}\left(x_{s}\right)
$$

where $K_{s}(\boldsymbol{\pi})$ is a function of probabilities and $v_{s}\left(x_{s}\right)$ is a function of $x_{s}$. Due to eqn. (37), $K_{s}(\boldsymbol{\pi})$ must be the same for all $s=1,2, \ldots, S$, and can be denoted $K(\boldsymbol{\pi})$. Therefore from (36), we have

$$
u_{s}^{\prime}\left(x_{s} ; \boldsymbol{\pi}\right)=K(\boldsymbol{\pi}) \pi_{s} v_{s}\left(x_{s}\right) \quad(s=1,2,3, \ldots, S) .
$$


Since when $k_{s}=1, x_{s}=f\left(x_{1}, 1\right)=x_{1}$, we must have $v_{1}=v_{s}=v$. Defining

$$
u\left(x_{s}\right)=\int v\left(x_{s}\right) d s
$$

it immediately follows that the given demands can be rationalized by a utility function that is ordinally equivalent to a state independent Expected Utility function as defined in eqn. (1). Finally, since

$$
f\left(x_{1}, k_{s}\right)=u^{\prime-1}\left(\frac{u^{\prime}\left(x_{1}\right)}{k_{s}}\right)
$$

is strictly increasing in $k_{s}, u$ is strictly concave.

The demand restriction $x_{s}=f\left(x_{1}, k_{s}\right)$ in Theorem 2 will be referred to as the $k$-test. It should be stressed that $f$ depends on prices and probabilities only through $k_{s}$.

It is natural to wonder why the $S=2$ case is not included in Theorem 2 . Before addressing this question, we provide an example which illustrates that when $S=2$ the $k$-test by itself is not sufficient if $U$ is not additively separable.

Example 2 Assume that

$$
U(\mathbf{x} ; \boldsymbol{\pi})=\left(\frac{\pi_{1} x_{1}}{\pi_{1}+2 \pi_{2}}+x_{2}\right) x_{1}^{\frac{\pi_{1}}{2 \pi_{2}}}
$$

which can be verified to be strictly increasing in each of its argument and strictly quasiconcave. Next we directly verify that the demand functions corresponding to this utility pass our $k$-test. The first order condition gives that

$$
\frac{\pi_{1}\left(x_{1}+x_{2}\right)}{2 \pi_{2} x_{1}}=\frac{p_{1}}{p_{2}}
$$

implying that

$$
x_{2}=\left(2 k_{2}-1\right) x_{1},
$$

where

$$
k_{2}=\frac{\pi_{2} p_{1}}{\pi_{1} p_{2}} .
$$

Denote

$$
f\left(x_{1}, k_{2}\right)=\left(2 k_{2}-1\right) x_{1}
$$

It is clear that $x_{2}=f\left(x_{1}, k_{2}\right)$ where $f\left(x_{1}, k_{2}\right)$ is increasing in $k_{2}$ and $f(x, 1)=x$. Therefore the $k$-condition is satisfied. However, (46) is clearly not an Expected Utility. Therefore for the $S=2$ case, the Theorem $2 k$-test is not sufficient and is only valid as a necessary condition. 
To understand why the $k$-test fails for this example, consider the very different treatment of the $S=2$ and $S>2$ cases in Samuelson (1947, pp. 176-183) when discussing additive separability. When $S=2, U$ is (ordinally) additively separable if and only if

$$
\frac{\partial \ln \left(\frac{\partial U / \partial x_{1}}{\partial U / \partial x_{2}}\right)}{\partial x_{1} \partial x_{2}}=0
$$

When $S>2$, the necessary and sufficient conditions become (23) and (25) in Lemma 1. However when $S=2$, there is no way to derive (51) from Theorem 2. This can also be seen from the proof of Theorem 2. The first part of the proof shows that for $S>2$, the condition $x_{s}=f\left(x_{1}, k_{s}\right)$ implies additive separability (independent of whether probabilities vary or are fixed). The second part of the proof demonstrates that if this condition is satisfied for any $S$, the separable utility must be a state independent Expected Utility. Therefore, when $S=2$, Theorem 2 can only work as a necessary and not sufficient condition. Of course if one assumes that $U$ is additively separable (ruling out Example 2), then the conditions in Theorem 2 become necessary and sufficient for any $S$.

Remark 3 In contrast to our approach, Dybvig (1983, Theorem 10) assumes that $U$ is additively separable. He then shows that $U$ is a (state independent) Expected Utility function if and only if the following holds

$$
\left.\frac{\partial U / \partial x_{1}}{\partial U / \partial x_{s}}\right|_{x_{1}=x_{s}}=\frac{\pi_{1}}{\pi_{s}} .
$$

Therefore combining the results of Samuelson and Dybvig, one obtains another demand test for Expected Utility. It should be noted that Dybvig's test also assumes a standard contingent claim setting, where probabilities are fixed and treated as constants. This is a key difference between Dybvig's test and the k-test in Theorem 2, where probabilities are parameter values which are allowed to vary exogenously. It should be stressed that Theorem 2 requires probabilities and prices to enter into the relationship between $x_{1}$ and $x_{s}$ only through $k_{s}$ and this condition cannot be verified if the probabilities are fixed numbers. This important difference between our result and that of Dybvig can be illustrated using the exponential utility function (4) in Example 1. For this utility, it follows from the first order condition that for $s=2,3$,

$$
\frac{\pi_{1}\left(\exp \left(-\pi_{1} x_{1}\right)+\exp \left(-\pi_{2} x_{1}\right)+\exp \left(-\pi_{3} x_{1}\right)\right)}{\pi_{s}\left(\exp \left(-\pi_{1} x_{s}\right)+\exp \left(-\pi_{2} x_{s}\right)+\exp \left(-\pi_{3} x_{s}\right)\right)}=\frac{p_{1}}{p_{s}}
$$

or equivalently

$$
\frac{\exp \left(-\pi_{1} x_{1}\right)+\exp \left(-\pi_{2} x_{1}\right)+\exp \left(-\pi_{3} x_{1}\right)}{\exp \left(-\pi_{1} x_{s}\right)+\exp \left(-\pi_{2} x_{s}\right)+\exp \left(-\pi_{3} x_{s}\right)}=k_{s},
$$


where

$$
k_{s}=\frac{\pi_{s} p_{1}}{\pi_{1} p_{s}} .
$$

Since the left hand side of eqn. (54) depends on probabilities, it is impossible to have $x_{s}=f\left(x_{1}, k_{s}\right)$, where $f$ is a function independent of prices and probabilities. Therefore, the demands generated by this utility function cannot pass our test in Theorem 2. However, the demands generated by (4) will always pass Dybvig's test. To see this, first note that the utility function (4) is additively separable. Moreover, it follows from the first order condition that

$$
\frac{\partial U / \partial x_{1}}{\partial U / \partial x_{s}}=\frac{\pi_{1}\left(\exp \left(-\pi_{1} x_{1}\right)+\exp \left(-\pi_{2} x_{1}\right)+\exp \left(-\pi_{3} x_{1}\right)\right)}{\pi_{s}\left(\exp \left(-\pi_{1} x_{s}\right)+\exp \left(-\pi_{2} x_{s}\right)+\exp \left(-\pi_{3} x_{s}\right)\right)} .
$$

When evaluating the right hand side of the above equation at $x_{1}=x_{s}$, one can obtain

$$
\left.\frac{\partial U / \partial x_{1}}{\partial U / \partial x_{s}}\right|_{x_{1}=x_{s}}=\frac{\pi_{1}}{\pi_{s}}
$$

which satisfies Dybvig's condition.

We next consider an example which illustrates for the $S=2$ case the application of Theorem 2 as a necessary condition.

Example 3 Consider the following pair of demand functions

$$
x_{1}=\left(\frac{1}{p_{1}+p_{2}\left(\frac{\pi_{2} p_{1}}{\pi_{1} p_{2}}\right)^{2}}+\frac{\pi_{1}}{\left(\pi_{1}+\pi_{2}\right) p_{1}}\right) \frac{I}{2}
$$

and

$$
x_{2}=\left(\frac{\left(\frac{\pi_{2} p_{1}}{\pi_{1} p_{2}}\right)^{2}}{p_{1}+p_{2}\left(\frac{\pi_{2} p_{1}}{\pi_{1} p_{2}}\right)^{2}}+\frac{\pi_{2}}{\left(\pi_{1}+\pi_{2}\right) p_{2}}\right) \frac{I}{2} .
$$

Defining the component of the Slutsky matrix as $\sigma_{i j}(i, j=1,2)$ where

$$
\sigma_{i j}=\frac{\partial x_{i}}{\partial p_{j}}+x_{j} \frac{\partial x_{i}}{\partial I}
$$

it can be verified that

$$
\sigma_{12}=\sigma_{21}, \sigma_{11}<0, \sigma_{22}<0 \text { and } \sigma_{11} \sigma_{22}=\sigma_{12} \sigma_{21},
$$

implying that the Slutsky matrix is symmetric and negative semidefinite. Therefore, the given demands can be rationalized by a utility function. Following the Hurwicz-Uzawa integration process (Hurwicz and Uzawa 1971), we obtain

$$
U\left(x_{1}, x_{2} ; \pi_{1}, \pi_{2}\right)=\frac{m}{\pi_{1} \sqrt{\frac{p^{\frac{\pi_{2}+2 \pi_{1}}{\pi_{1}+\pi_{2}}}}{\pi_{1}^{2}+p \pi_{2}^{2}}}},
$$


where the relative price $p=p_{1} / p_{2}$ is given by

$$
p=\frac{\pi_{1}\left(\pi_{2} x_{2}-\pi_{1} x_{1}\right)+\sqrt{\pi_{1}^{2}\left(\pi_{1}^{2} x_{1}^{2}+\pi_{2}^{2} x_{2}^{2}+2 x_{1} x_{2}\left(4 \pi_{1}^{2}+4 \pi_{2}^{2}+9 \pi_{1} \pi_{2}\right)\right)}}{2 x_{1} \pi_{2}\left(\pi_{1}+2 \pi_{2}\right)}
$$

and the normalized income $m=I / p_{2}$ is given by

$$
m=\frac{\pi_{1}\left(\pi_{2} x_{2}-\pi_{1} x_{1}\right)+\sqrt{\pi_{1}^{2}\left(\pi_{1}^{2} x_{1}^{2}+\pi_{2}^{2} x_{2}^{2}+2 x_{1} x_{2}\left(4 \pi_{1}^{2}+4 \pi_{2}^{2}+9 \pi_{1} \pi_{2}\right)\right)}}{2 \pi_{2}\left(\pi_{1}+2 \pi_{2}\right)}+x_{2} .
$$

The above form of utility (62) is clearly not additively separable and does not take the Expected Utility form. This is confirmed by the following application of the $k$-test from Theorem 2. Noticing that

$$
\frac{x_{2}}{x_{1}}=\frac{\frac{1}{p_{1}+p_{2}\left(\frac{\pi_{2} p_{1}}{\pi_{1} p_{2}}\right)^{2}}+\frac{\pi_{1}}{\left(\pi_{1}+\pi_{2}\right) p_{1}}}{\frac{\left(\frac{\pi_{2} p_{1}}{\pi_{1} p_{2}}\right)^{2}}{p_{1}+p_{2}\left(\frac{\pi_{2} p_{1}}{\pi_{1} p_{2}}\right)^{2}}+\frac{\pi_{2}}{\left(\pi_{1}+\pi_{2}\right) p_{2}}}
$$

is not solely a function of $k_{2}=\frac{\pi_{2} p_{1}}{\pi_{1} p_{2}}$, it follows from Theorem 2 that the demand functions (58)-(59) cannot be rationalized by an Expected Utility representation for all values of $x_{1}$ and $x_{2}$.

\subsection{Demand Derivative Restrictions}

In the prior subsection, we provide a characterization that is necessary and sufficient for demands to be rationalizable by a state independent Expected Utility. To ensure that the given functions are actually demand functions (i.e., can be rationalized by some utility function), we have assumed that the Slutsky symmetry and negative semidefiniteness conditions are satisfied. But what additional conditions does the assumption of Expected Utility impose on the Slutsky matrix? Goldman and Uzawa (1964) provide a partial answer - they show that the assumption of separable utility can be tested with a condition on the Slutsky terms. We simplify their condition in our setting and provide additional conditions which collectively are necessary and sufficient for Expected Utility maximization. Our conditions involve the partial derivatives of the assumed demand with respect to probabilities.

It will prove useful to derive the Slutsky equation using the implicit function theorem (see, for example, Kreps 2012). From this it will be straightforward to derive the matrix of partial derivatives of demand with respect to probabilities given by eqn. (72) below. 
The solution to the optimization problem (3) is determined by $F(\mathbf{x}, \lambda, \mathbf{p}, I, \boldsymbol{\pi})=$ 0 where

$$
F(\mathbf{x}, \lambda, \mathbf{p}, I, \boldsymbol{\pi})=\left\{\begin{array}{l}
D_{\mathbf{x}} U(\mathbf{x} ; \boldsymbol{\pi})-\lambda \mathbf{p}=\mathbf{0} \\
I-\mathbf{p} \cdot \mathbf{x}=0
\end{array},\right.
$$

and $\lambda$ is the Lagrange multiplier. The utility $U$ is assumed to be differentiably strictly concave. It follows that $D_{\mathbf{x x}}^{2} U(\mathbf{x} ; \boldsymbol{\pi})$ is negative definite which, as is wellknown (see Kreps 2012, Proposition 11.10), implies that $D_{\mathbf{x}, \lambda} F(\mathbf{x}, \lambda, \mathbf{p}, I, \boldsymbol{\pi})$ is invertible and the implicit function theorem can be applied. We can define

$$
\left(\begin{array}{cc}
\mathcal{K} & -\boldsymbol{\tau} \\
-\boldsymbol{\tau}^{T} & b
\end{array}\right)=\left(\begin{array}{cc}
D_{\mathbf{x x}}^{2} U(\mathbf{x} ; \boldsymbol{\pi}) & -\mathbf{p} \\
-\mathbf{p}^{T} & 0
\end{array}\right)^{-1}
$$

where $\mathcal{K}$ is a symmetric $S \times S$ matrix and $\boldsymbol{\tau}$ is a $S \times 1$ vector. Defining

$$
\Sigma=\lambda \mathcal{K}
$$

and then applying the implicit function theorem yields

$$
D_{\mathbf{p}, I, \boldsymbol{\pi}}(\mathbf{x}, \lambda)=-\left(D_{\mathbf{x}, \lambda} F\right)^{-1} D_{\mathbf{p}, I, \boldsymbol{\pi}} F
$$

Therefore we have

$$
\begin{aligned}
D_{\mathbf{p}} \mathbf{x} & =\Sigma-\boldsymbol{\tau} \mathbf{x}^{T} \\
D_{I} \mathbf{x} & =\boldsymbol{\tau} \\
D_{\boldsymbol{\pi}} \mathbf{x} & =-\mathcal{K} D_{\boldsymbol{\pi} \mathbf{x}}^{2} U(\mathbf{x} ; \boldsymbol{\pi}) .
\end{aligned}
$$

It is well-known that the symmetric matrix $\Sigma=\left(\sigma_{i j}\right)_{S \times S}$ has rank $S-1$ with $\Sigma \mathbf{p}=\mathbf{0}$.

We have the following theorem.

Theorem 3 When $S>2$, demand is rationalized by a state independent Expected Utility function of the form (1) if and only if the following conditions hold

$$
\begin{gathered}
\frac{\frac{\partial x_{1}}{\partial p_{j}}}{\tau_{1}}=\frac{\frac{\partial x_{i}}{\partial p_{j}}}{\tau_{i}} \quad \forall i, j \in\{2,3, \ldots, S\} \text { and } i \neq j, \\
\frac{\partial x_{j}}{\partial \pi_{s}}=-\sigma_{j s} \frac{p_{s}}{\pi_{s}} \quad \forall j, s \in\{1,2, \ldots, S\}
\end{gathered}
$$

and

$$
x_{i}=x_{j} \quad \text { only if } \frac{p_{i} / \pi_{i}}{p_{j} / \pi_{j}}=1 \quad \forall i, j \in\{1,2, \ldots, S\}
$$


Before proving the theorem it is useful to note that (73) is equivalent to the following condition on $\sigma_{i j}$,

$$
\frac{\sigma_{1 j}}{\tau_{1}}=\frac{\sigma_{i j}}{\tau_{i}} \forall i, j \in\{2,3, \ldots, S\} \text { and } i \neq j
$$

and that (74) implies that demand is homogenous of degree zero in probabilities, i.e., we have

$$
\sum_{s=1}^{S} \frac{\partial x_{j}}{\partial \pi_{s}} \pi_{s}=0 .
$$

To prove the theorem first note that Theorem 4 in Goldman and Uzawa (1964) implies the following lemma.

Lemma 2 Demand is rationalizable by a separable utility function

$$
U(\mathbf{x} ; \boldsymbol{\pi})=\sum_{s=1}^{S} u_{s}\left(x_{s} ; \boldsymbol{\pi}\right)
$$

if and only if eqn. (76) holds.

Since Goldman and Uzawa (1964) consider a more general setting, their condition is actually more complicated. They require that for all $i \neq j$ and all $k \neq l$

$$
\frac{\sigma_{i j}}{\tau_{i} \tau_{j}}=\frac{\sigma_{k l}}{\tau_{k} \tau_{l}} .
$$

However, note that eqn. (79) is equivalent to (76) together with the following

$$
\frac{\sigma_{1 s}}{\tau_{s}}=\frac{\sigma_{1 k}}{\tau_{k}} .
$$

In our setting with only one good per state, this latter equation, (80), is redundant since it follows from (76) by Slutsky symmetry. The fact that for all $s, k, \sigma_{k s}=\sigma_{s k}$ implies that $\frac{\sigma_{1 s}}{\tau_{s}}=\frac{\sigma_{k s}}{\tau_{k}}=\frac{\sigma_{s k}}{\tau_{k}}=\frac{\sigma_{1 k}}{\tau_{k}}$.

We are now in a position to prove the theorem.

Proof. To see the necessity of (73)-(75), note that under the assumption of state independent Expected Utility, eqn. (72) simplifies to

$$
D_{\pi} \mathbf{x}=-\mathcal{K} \operatorname{diag}\left(u^{\prime}\left(x_{s}\right)\right)=-\Sigma \operatorname{diag}\left(\frac{p_{s}}{\pi_{s}}\right),
$$

where diag denotes the diagonal matrix. The second equality in (81) follows from the definition of $\Sigma$ and the fact that the agent's first order conditions imply that for all $s=1, \ldots, S, u^{\prime}\left(x_{s}\right)=\lambda \frac{p_{s}}{\pi_{s}}$. Therefore (81) implies (74) while (73) follows from Lemma 2. Conditions (75) are obvious. 
To prove sufficiency, first note that following Lemma 2 condition (76) implies the existence of a utility function of the form

$$
U(\mathbf{x} ; \boldsymbol{\pi})=\sum_{s=1}^{S} u_{s}\left(x_{s} ; \boldsymbol{\pi}\right) .
$$

Conditions (74) uniquely determine the matrix $D_{\boldsymbol{\pi}} \mathbf{x}(\mathbf{p}, I, \boldsymbol{\pi})$. Since $\Sigma$ has rank $S-1$ and $\Sigma \mathbf{x}=\mathbf{0}$ only if $\mathbf{x}=\alpha \mathbf{p}$ for some $\alpha$ all solutions to eqn. (74) must have the form for each $s, j \in\{1, \ldots, S\}$,

$$
\frac{\partial^{2} u_{s}}{\partial \pi_{j} \partial x_{s}}=\alpha_{j} p_{s} \text { if } j \neq s ; \quad \frac{\partial^{2} u_{s}}{\partial \pi_{j} \partial x_{s}}=\alpha_{s} p_{s}+\lambda \frac{p_{s}}{\pi_{s}} \text { if } j=s,
$$

for some $\alpha_{1}, \ldots, \alpha_{S}$, where $\lambda$ denotes, as above, the Lagrange multiplier. Noticing that

$$
\frac{\partial^{2}\left(\frac{u_{s}}{\pi_{s}}\right)}{\partial \pi_{s} \partial x_{s}}=\frac{1}{\pi_{s}} \frac{\partial^{2} u_{s}}{\partial \pi_{s} \partial x_{s}}-\frac{1}{\pi_{s}^{2}} \frac{\partial u_{s}}{\partial x_{s}}
$$

and

$$
\frac{\partial u_{s}}{\partial x_{s}}=\lambda p_{s}
$$

we can rewrite (83) as

$$
\frac{\partial^{2}\left(\frac{u_{s}}{\pi_{s}}\right)}{\partial \pi_{j} \partial x_{s}}=\frac{\alpha_{j} p_{s}}{\pi_{s}} \forall j, s \in\{1,2, \ldots, S\} .
$$

Without loss of generality, assume that $\alpha_{k} \neq 0(\forall k \in\{1,2, \ldots, S\}) .{ }^{4}$ Then for all $i \neq j \in\{1,2, \ldots, S\}$, we have

$$
\frac{\frac{\partial^{2}\left(u_{i} / \pi_{i}\right)}{\partial x_{i} \partial \pi_{k}}}{\frac{\partial^{2}\left(u_{j} / \pi_{j}\right)}{\partial x_{j} \partial \pi_{k}}}=\frac{\pi_{j} p_{i}}{\pi_{i} p_{j}} \quad \forall k \in\{1,2, \ldots, S\} .
$$

But the first order conditions for optimality imply that

$$
\frac{p_{i}}{p_{j}}=\frac{\frac{\partial u_{i}}{\partial x_{i}}}{\frac{\partial u_{j}}{\partial x_{j}}} \Leftrightarrow \frac{\frac{\partial\left(u_{i} / \pi_{i}\right)}{\partial x_{i}}}{\frac{\partial\left(u_{j} / \pi_{j}\right)}{\partial x_{j}}}=\frac{\pi_{j} p_{i}}{\pi_{i} p_{j}}
$$

and hence we obtain

$$
\frac{\frac{\partial^{2}\left(u_{i} / \pi_{i}\right)}{\partial x_{i} \partial \pi_{k}}}{\frac{\partial^{2}\left(u_{j} / \pi_{j}\right)}{\partial x_{j} \partial \pi_{k}}}=\frac{\frac{\partial\left(u_{i} / \pi_{i}\right)}{\partial x_{i}}}{\frac{\partial\left(u_{j} / \pi_{j}\right)}{\partial x_{j}}} .
$$

Denoting

$$
h_{s}\left(x_{s} ; \boldsymbol{\pi}\right)=\frac{\partial\left(u_{s} / \pi_{s}\right)}{\partial x_{s}}(s=i, j),
$$

\footnotetext{
${ }^{4}$ If $\alpha_{k}=0$ for some $k$, one can always multiply the utility function with some nonzero function of $\pi_{k}$ to make $\alpha_{k} \neq 0$.
} 
we have

$$
\frac{\partial\left(\ln h_{i}\left(x_{i} ; \boldsymbol{\pi}\right)\right)}{\partial \boldsymbol{\pi}}=\frac{\partial\left(\ln h_{j}\left(x_{j} ; \boldsymbol{\pi}\right)\right)}{\partial \boldsymbol{\pi}},
$$

where it follows from the additive separability of $U$ that $h_{s}$ must be a function of only one $x_{s}(s=i, j)$. Following the proof of Theorem 2 ,

$$
h_{s}\left(x_{s} ; \boldsymbol{\pi}\right)=K_{s}(\boldsymbol{\pi}) v_{s}\left(x_{s}\right) \quad(s=i, j),
$$

where $K_{s}$ and $v_{s}$ are defined in (41). Finally, it follows from the first order condition that

$$
\frac{K_{i}(\boldsymbol{\pi}) v_{i}\left(x_{i}\right)}{K_{j}(\boldsymbol{\pi}) v_{j}\left(x_{j}\right)}=\frac{\pi_{j} p_{i}}{\pi_{i} p_{j}}
$$

Condition (75) implies that for all $x$ and $i \neq j \in\{1,2, \ldots, S\}$,

$$
\frac{K_{i}(\boldsymbol{\pi}) v_{i}(x)}{K_{j}(\boldsymbol{\pi}) v_{j}(x)}=1
$$

and hence $K_{1}(\boldsymbol{\pi}) v_{1}(x)=K_{s}(\boldsymbol{\pi}) v_{s}(x)$ for all $s$. Without loss of generality, taking

$$
K_{s}(\boldsymbol{\pi})=K(\boldsymbol{\pi}) \text { and } u\left(x_{s}\right)=\int v_{s}\left(x_{s}\right) d s=\int v\left(x_{s}\right) d s \quad(\forall s=1,2, \ldots, S),
$$

it immediately follows that the given demands can be rationalized by a utility function that is ordinally equivalent to a state independent Expected Utility function as defined in eqn. (1), which concludes the proof.

Remark 4 It should be noted that additive separability plays a crucial role in the above proof. Without additive separability, it can be verified that for the utility function in Example 2, we have

$$
\begin{gathered}
\frac{\partial x_{1}}{\partial \pi_{1}}=\frac{2 \pi_{2} p_{1} I}{\left(\pi_{1} p_{2}-\left(\pi_{1}+2 \pi_{2}\right) p_{1}\right)^{2}}, \\
\frac{\partial x_{2}}{\partial \pi_{1}}=-\frac{2 \pi_{2} p_{1}^{2} I}{p_{2}\left(\pi_{1} p_{2}-\left(\pi_{1}+2 \pi_{2}\right) p_{1}\right)^{2}}, \\
\sigma_{11}=-\frac{2 \pi_{1} \pi_{2} I}{\left(\pi_{1} p_{2}-\left(\pi_{1}+2 \pi_{2}\right) p_{1}\right)^{2}}, \\
\sigma_{21}=\frac{2 \pi_{1} \pi_{2} p_{1} I}{p_{2}\left(\pi_{1} p_{2}-\left(\pi_{1}+2 \pi_{2}\right) p_{1}\right)^{2}},
\end{gathered}
$$

implying that

$$
\frac{\partial x_{1}}{\partial \pi_{1}}=-\sigma_{11} \frac{p_{1}}{\pi_{1}} \text { and } \frac{\partial x_{2}}{\partial \pi_{1}}=-\sigma_{21} \frac{p_{1}}{\pi_{1}}
$$

Similarly, we can verify that

$$
\frac{\partial x_{1}}{\partial \pi_{2}}=-\sigma_{12} \frac{p_{2}}{\pi_{2}} \text { and } \frac{\partial x_{2}}{\partial \pi_{2}}=-\sigma_{22} \frac{p_{2}}{\pi_{2}} .
$$

Therefore, (74) holds. However, the utility function in Example 2 is clearly not an Expected Utility. 


\section{References}

Afriat, Sydney N. 1967. "The Construction of a Utility Function from Expenditure Date." International Economic Review 8: 67-77.

Andreoni, James and Charles Sprenger. 2012. "Risk Preferences Are Not Time Preferences." American Economic Review 102: 3357-3376.

Antonelli, Giovanni B. 1886. "On the Mathematical Theory of Political Economy." in Preferences, Utility, and Demand: A Minnesota Symposium. ed. by John. S. Chipman, Leonid Hurwicz, Marcel K. Richter, Hugo F. Sonnenschein. New York: Harcourt Brace Jovanavich Ltd.

Chiappori, Pierre-André and Jean-Charles Rochet. 1987. "Revealed Preferences and Differentiable Demand." Econometrica 55: 687-691.

Choi, Syngjoo, Raymond Fisman, Douglas Gale and Shachar Kariv. 2007. "Consistency and Heterogeneity of Individual Behavior under Uncertainty." American Economic Review 97: 1921-1938.

Dybvig, Philip H. 1983. "Recovering Additive Utility Functions." International Economic Review 24: 379-396.

Echenique, Federico and Kota Saito. 2013. "Savage in the Market." Unpublished Working Paper.

Green, Richard C. and Sanjay Srivastava. 1986. "Expected Utility Maximization and Demand Behavior." Journal of Economic Theory 38: 313-323.

Goldman, Steven M. and Hirofumi Uzawa. 1964. "A Note on Separability in Demand Analysis." Econometrica 32: 387-398.

Hurwicz, Leonid and Hirofumi Uzawa. 1971. "On the Integrability of Demand Functions." in Preferences, Utility, and Demand: A Minnesota Symposium. ed. by John. S. Chipman, Leonid Hurwicz, Marcel K. Richter, Hugo F. Sonnenschein. New York: Harcourt Brace Jovanavich Ltd.

Kreps, David M. 2012. Microeconomic Foundations I: Choice and Competitive Markets. Princeton: Princeton University Press.

Kubler, Felix and Karl Schmedders. 2010. "Non-parametric Counterfactual Analysis in Dynamic General Equilibrium." Economic Theory 25: 181-200. 
Polisson, Matthew and John Quah. 2013. "Revealed Preference Tests under Risk and Uncertainty." University of Leicester Working Paper No. 13/24.

Samuelson, Paul A. 1938. "A Note on the Pure Theory of Consumer Behavior." Economica 5: 61-71.

— 1947. Foundations of Economic Analysis. Cambridge: Harvard University Press.

Slutsky, Eugen 1915. "On the Theory of the Budget of the Consumer." in Readings in Price Theory. ed. by American Economic Association, G. J. Stigler, 27-56. Varian, Hal R. 1982. "The Nonparametric Approach to Demand Analysis." Econometrica 50: 945-973.

— 1983. "Nonparametric Tests of Models of Investor Behavior." Journal of Financial and Quantitative Analysis 18: 269-278.

— 1988. "Estimating Risk Aversion from Arrow-Debreu Portfolio Choice." Econometrica 56: 973-979. 